Wijngaard, C.C. van den, Dijkstra, F., Pelt, W. van, Asten, L. van, Kretzschmar, M., Schimmer, B., Nagelkerke, N.J.D., Vellema, P., Donker, G.A., Koopmans, M.P.G. In search of hidden Q-fever outbreaks: linking syndromic hospital clusters to infected goat farms. Epidemiology and Infection: 2011, 139(1), 19-26

\begin{tabular}{|l|l|}
\hline Postprint Version & 1.0 \\
\hline Journal website & $\underline{\mathrm{http}: / / \text { journals.cambridge.org/action/displayAbstract?fromPage=online\&aid=77 }}$ \\
\hline Pubmed link & $\underline{\underline{57576}}$ \\
\hline DOI & $\underline{\mathrm{htp}: / / \text { www.ncbi.nlm.nih.gov/pubmed/20478085 }}$ \\
\hline
\end{tabular}

This is a NIVEL certified Post Print, more info at http://www.nivel.eu

\title{
In search of hidden Q-fever outbreaks: linking syndromic hospital clusters to infected goat farms
}

\author{
C. C. VAN DEN WIJNGAARD ${ }^{\mathrm{A1}} \mathrm{c1}$, F. DIJKSTRA ${ }^{\mathrm{A} 1}$, W. VAN PELT ${ }^{\mathrm{A} 1}, \mathrm{~L}$. VAN ASTEN ${ }^{\mathrm{A} 1}, \mathrm{M}$. \\ KRETZSCHMAR $^{\mathrm{A} 1 \mathrm{~A} 2}$, B. SCHIMMER ${ }^{\mathrm{A} 1}$, N. J. D. NAGELKERKE ${ }^{\mathrm{A} 3}$, P. VELLEMA ${ }^{\mathrm{A} 4}$, G. A. DONKER $^{\mathrm{A} 5}$ \\ AND M. P. G. KOOPMANS ${ }^{\mathrm{A} 1 \mathrm{~A} 6}$ \\ ${ }^{a 1}$ National Institute for Public Health and the Environment, Bilthoven, The Netherlands \\ a2 Julius Centre for Health Sciences and Primary Care, University Medical Centre Utrecht, The Netherlands \\ ${ }^{a 3}$ United Arab Emirates University, Al-Ain, United Arab Emirates \\ ${ }^{a 4}$ Animal Health Service, Deventer, The Netherlands \\ ${ }^{a 5}$ Nivel, Netherlands Institute of Health Services Research, Utrecht, The Netherlands \\ ${ }^{a 6}$ Erasmus Medical Center, Rotterdam, The Netherlands
}

\begin{abstract}
SUMMARY
Large Q-fever outbreaks were reported in The Netherlands from May 2007 to 2009, with dairy-goat farms as the putative source. Since Q-fever outbreaks at such farms were first reported in 2005, we explored whether there was evidence of human outbreaks before May 2007. Space-time scan statistics were used to look for clusters of lower-respiratory infections (LRIs), hepatitis, and/or endocarditis in hospitalizations, 2005-2007. We assessed whether these were plausibly caused by Q fever, using patients' age, discharge diagnoses, indications for other causes, and overlap with reported Q fever in goats/humans. For seven detected LRI clusters and one hepatitis cluster, we considered Q fever a plausible cause. One of these clusters reflected the recognized May 2007 outbreak. Real-time syndromic surveillance would have detected four of the other clusters in 2007, one in 2006 and two in 2005, which might have resulted in detection of Q-fever outbreaks up to 2 years earlier.
\end{abstract}

\section{INTRODUCTION}

Q fever is a zoonosis occurring worldwide caused by Coxiella burnetii, an intracellular bacterium. Although most human infections remain asymptomatic or present as a non-specific flu-like illness, severe acute Q fever presents primarily with atypical pneumonia or hepatitis. The infection poses an increased risk for pregnant women and persons with heart-valve disorders or impaired immunity, who may develop chronic disease with endocarditis as its most frequent chronic clinical manifestation. Q fever in pregnancy, whether symptomatic or asymptomatic, may result in adverse pregnancy outcomes. Cattle, sheep and particularly goats are considered the primary reservoirs from which human infection occurs, typically by inhalation of infected aerosols, and less commonly through ingestion [1-3].

For many years, Q fever in humans was very rare in The Netherlands, with around 15 reported cases per year [4], but since the end of May 2007, outbreaks have occurred in rural areas mainly in the southern part of the country, with 20-25\% of the reported cases requiring hospitalization [5]. In 2007, 178 Q-fever cases were reported, most of which occurred in May and June 2007. In total, 31\% $(n=55)$ of these cases occurred 
Wijngaard, C.C. van den, Dijkstra, F., Pelt, W. van, Asten, L. van, Kretzschmar, M., Schimmer, B., Nagelkerke, N.J.D., Vellema, P., Donker, G.A., Koopmans, M.P.G. In search of hidden Q-fever outbreaks: linking syndromic hospital clusters to infected goat farms. Epidemiology and Infection: 2011, 139(1), 19-26

in a relatively small rural area [6]. In 2008 and 2009, large outbreaks of Q fever recurred with increasing numbers of reported cases and an expanding geographic area [5, 7].

Dairy-goat farms are considered the most likely source of infection for these outbreaks, although evidence is still inconclusive [5, 7, 8]. Q-fever abortion waves have been reported at several dairy-goat farms, starting at least 2 years before the first recognized human outbreak [5, 9]. This time lag raised the question whether unrecognized human outbreaks may have preceded May 2007, particularly since most severe cases present as pneumonia, for which laboratory tests are often not requested. In fact, the diagnoses of the May/June 2007 outbreak cases were delayed by several weeks, until pneumonia patients were retested for C. burnetii, triggered by an increase in the number of $\mathrm{Q}$ fever reports in humans in the region $[8,10]$. Syndromic surveillance allows monitoring of clinical syndromes such as 'pneumonia' or 'lowerrespiratory infection' (LRI) independent of laboratory confirmation. It also permits detection of outbreaks of diseases for which diagnostics are either not available or not requested. In order to optimize its sensitivity for detection of local outbreaks, surveillance of syndrome spikes can be performed in space and time $[11,12]$. Earlier we demonstrated the value of this approach by showing that syndromic surveillance of Dutch hospitalizations can indeed detect local lower-respiratory disease outbreaks [13].

In the current study, we retrospectively explored whether there is evidence for human Q-fever outbreaks in The Netherlands in 2005-2007, before the May/June 2007 outbreak. Using space-time scan statistics, we looked for local increased numbers of hospitalized patients with LRIs and other syndromes that can be associated with $C$. burnetii infection. Based on available epidemiological and surveillance data, and the geographical proximity of small-ruminant farms that tested positive for $\mathrm{Q}$ fever, we then assessed whether these local increases could have been caused by $\mathrm{Q}$ fever or by other infections like respiratory syncytial virus (RSV) or influenza. Finally, we evaluated whether real-time syndromic surveillance of hospitalizations could have accelerated the detection of human Q-fever outbreaks.

\section{METHODS}

\section{Hospitalization data}

For the period 1 January 2005 to 30 September 2007, hospitalization data were obtained from the Dutch National Medical Register, which has about $80 \%$ population coverage. We excluded data before 2005, as data on farms that tested positive for $\mathrm{Q}$ fever was not then available. The data included discharge and secondary diagnoses by date of hospitalization. We selected all hospitalization records showing diagnoses involving clinical syndromes compatible with Q fever, i.e. LRI, hepatitis, and/or endocarditis. We used a case definition for LRI, which has proved functional for detecting severe respiratory disease outbreaks in hospitalization data [13]. See Appendix A (available online) for detailed hepatitis and endocarditis case definitions.

We analysed 108338 hospitalizations for LRI after excluding 5\% of 114245 records because of patients readmitted within six consecutive weeks; 3826 hospitalizations for hepatitis after excluding $29 \%$ of 5382 records because of readmissions from 1999 to 2007 (to avoid chronic cases first hospitalized before 2005); and 2130 hospitalizations for endocarditis after excluding 18\% of 2612 records because of readmissions from 1999 to 2007 (to avoid chronic cases first hospitalized before 2005). Data were aggregated by week of

hospitalization, postal code, and patients' age group (0-4, 5-19, 20-49, 50-64, 65 years). Of these, we regarded the 20-49 and 50-64 years age groups to be at higher risk for Q fever, since most reported cases were adults, with the median age $\sim 50$ years [5, 7]. Since higher levels of spatial resolution can result in more sensitive detection of outbreaks [14, 15], we used 4-digit postal codes designating 4023 areas in a 16.3 million population. This provided the highest level of spatial resolution available within national privacy regulations.

\section{Infectious disease surveillance data}

To assess alternative causes (other than $\mathrm{Q}$ fever) for detected local clusters of syndromic hospital cases, we explored mandatory reports of psittacosis, Legionnaires' disease, hepatitis A, B and C, and Q fever, although prior to 2007 Q-fever cases may have remained undetected or misdiagnosed. The counts of reports were aggregated by week of disease onset and 4-digit postal codes. We used regional data on influenza-like illness (ILI) to assess whether a rise in local LRI cases could be due to influenza. These data are collected weekly by a sentinel network of general practitioners, the Continuous Morbidity Registration (CMR) 
Wijngaard, C.C. van den, Dijkstra, F., Pelt, W. van, Asten, L. van, Kretzschmar, M., Schimmer, B., Nagelkerke, N.J.D., Vellema, P., Donker, G.A., Koopmans, M.P.G. In search of hidden Q-fever outbreaks: linking syndromic hospital clusters to infected goat farms. Epidemiology and Infection: 2011, 139(1), 19-26

centres, which provide $1 \%$ population coverage, representative by age, gender, geographic distribution and population density [16]. ILI is defined as an acute beginning of a respiratory infection with fever (rectal

temperature $\quad 38^{\circ} \mathrm{C}$ ), and with at least one of the following symptoms present: cough, coryza, sore throat, frontal headache, retrosternal pain, myalgia. Due to the limited number of sentinel practices, these weekly data were aggregated into four major regions instead of 4-digit postal codes, as for the hospitalization data.

\section{Data on positive goat and sheep farms}

To identify possible point sources of C. burnetii, we used Q-fever-related abortion waves on dairy-goat and dairy-sheep farms as reported to the Dutch Animal Health Service. For each farm that tested positive for C. burnetii between 2005 and 2008, we recorded its 4-digit postal code and the date of its first submission of placental tissue.

Diagnostic tests for Q fever as a cause of abortion waves in animals were first available in 2004. In 2005 the first positive results were obtained from two dairy-goat farms, in 2006 from six dairy-goat farms and one dairy-sheep farm, in 2007 from seven dairy-goat farms, and in 2008 from eight dairy-goat farms and one dairy-sheep farm. Until 12 June 2008, reporting of abortion waves and subsequent laboratory testing of placental tissue were voluntary in The Netherlands; thus previous Q-fever abortion waves cannot be excluded in areas making no reports. For one farm, placental tissue from 2001 was retrospectively tested and found positive for C. burnetii.

We regarded these farms as possible point sources of $C$. burnetii during the entire study period, as some were known to have had abortion problems in the year before and/or after they first submitted placental tissue. Moreover, infected animals can become long-term shedders, and C. burnetii is very persistent in the environment [17].

\section{Scan statistics for space-time clusters}

In order to detect a possible increase in Q-fever-related regional cases in the hospitalization data, we used a space-time permutation scan statistic that compared observed and expected numbers of cases in flexible circular areas over flexible time periods [18]. SaTScan software and SAS (version 9.1, SAS Institute Inc., USA) were used to run the scan-statistic [19, 20].

For each week in the analysis period, we ran the scan statistic on data from the preceding year, thus simulating prospective (real-time) surveillance. This generated weekly space-time signals. To indicate the significance of detected space-time signals, we used recurrence intervals, which indicate how often a signal of the observed significance would be observed by chance under the hypothesis of no outbreak [21]. That is, if the recurrence interval of a signal is say 1 year, one signal of the observed significance is expected in 1

year. If the recurrence interval of a signal was 1 year, it was viewed as a significant signal. Besides that, we also used a threshold of recurrence intervals 5 years to indicate highly significant signals. We defined a cluster as a group of successive overlapping space-time signals, since overlap suggests the same cause; we defined a cluster signal as an individual space-time signal within a cluster. Unlike an earlier study [13], in the present study we also assessed whether the same cause for successive overlapping cluster signals seemed unlikely due to shifts in space or time windows or shifts in predominant age groups or discharge diagnoses. We used previously validated parameter settings for the scan statistic, with minimal constraints on the maximum space and/or time windows, to avoid incorrect assumptions about time, geographical location, or size of possible Q-fever outbreaks [13].

We also performed space-time scans on specific infectious disease data (mandatory reports and ILI sentinel data), to assess whether these might explain the space-time clusters of hospitalizations that we detected. For the mandatory reports, the same parameter settings were used as for the hospitalizations. For the ILI data, the space-time scans were performed using both case data and population-at-risk data [22], as previously described [13].

\section{LRI clusters}

To determine the LRI clusters for which Q fever was an unlikely, possible or plausible cause, we used two-step criteria (Fig. 1). In step 1, the upper box shows the criteria for LRI clusters unlikely to be caused by $\mathrm{Q}$ fever, based on indications for other causative pathogens and epidemiological characteristics (e.g. patients' age) that differed from the confirmed Q-fever cases. The lower box shows the criteria for LRI 
Wijngaard, C.C. van den, Dijkstra, F., Pelt, W. van, Asten, L. van, Kretzschmar, M., Schimmer, B., Nagelkerke, N.J.D., Vellema, P., Donker, G.A., Koopmans, M.P.G. In search of hidden Q-fever outbreaks: linking syndromic hospital clusters to infected goat farms. Epidemiology and Infection: 2011, 139(1), 19-26

clusters possibly caused by Q fever, based on epidemiological data [5, 7, 23] as well as overlap with clusters of human Q-fever reports. In step 2, we assessed whether clusters identified as possibly caused by Q fever (in step 1) showed geographical overlap with farms that tested positive in 2005-2008. If so, we considered Q fever a plausible cause for these clusters.

\section{[FIGURE 1]}

\section{Hepatitis and endocarditis clusters}

To assess whether detected hepatitis and/or endocarditis clusters might have been caused by Q fever, we again used two-step criteria, similar as for the LRI clusters. In step 1, we now evaluated whether hepatitis clusters overlapped with clusters of hepatitis A, B, or C reports and assessed whether hepatitis and/or endocarditis clusters had any characteristics (age distribution, specific diagnoses) suggesting causes other than Q fever. In step 2, clusters for which no alternative causes were found, were assessed for overlap with reported Q-fever abortion waves at farms in 2005-2008. If they overlapped, we considered Q fever a plausible cause for these human disease clusters.

The patients of all clusters for which we considered Q fever a plausible cause were then line-listed and inspected for data anomalies that might have caused a cluster signal. Finally, we evaluated to what extent actual prospective syndromic surveillance could have accelerated the detection of human Q-fever outbreaks.

\section{RESULTS}

\section{LRI clusters}

We detected 20 LRI clusters in hospitalization data between 2005 and 2007 [for details see Appendix B, Table B1 (available online)]. Applying the criteria in Figure 1, for seven of these LRI clusters, Q fever was considered a plausible cause; for three clusters a possible cause, and for ten clusters an unlikely cause. Figure 2 shows all LRI clusters and signals on a timescale, and Figure $3 a$ shows the locations of the LRI clusters for which we considered Q fever a plausible cause.

\section{[FIGURE 2][FIGURE 3]}

\section{Q fever: a plausible cause}

Of the seven clusters for which Q fever was considered a plausible cause, one reflected the known May/June 2007 outbreak, as it strongly overlapped in space and time with that outbreak. If monitored in real time, this cluster would have given the first signal at the end of May 2007, i.e. in the same week that suspicious pneumonia patients were first reported, and 6 weeks before the first positive laboratory results for $C$. burnetii were obtained $[8,10]$.

Of the other six clusters, three would have given the first signal 1-2 years earlier than the known outbreak in 2007 (two in 2005 and one in 2006), one cluster 3 months earlier, one at the same time, and one cluster 4 months afterwards [Fig. 2 and Appendix B, Table B1 (online)]. All but one of these clusters occurred in the southeast or the middle of the country (Fig. 3 a) but in a more widespread area than the May/June 2007 outbreak. The two 2005 clusters and two clusters in 2007 were highly significant with recurrence intervals 5 years.

\section{Q fever: a possible cause}

For only three clusters was $\mathrm{Q}$ fever a possible cause, i.e. not plausible because they lacked geographical proximity to Q-fever abortion waves on farms. All three clusters occurred in 2007 [see Fig. 2 and Appendix B (online)].

\section{$Q$ fever: an unlikely cause}

The ten clusters for which $\mathrm{Q}$ fever was considered an unlikely cause were not assessed for geographical overlap with infected farms, because other pathogens (e.g. influenza and RSV) seemed probable. As previously described, one cluster was clearly due to Legionella, as it comprised many cases of Legionnaires' disease and overlapped in space and time with a known Legionella outbreak [13, 24]. 
Wijngaard, C.C. van den, Dijkstra, F., Pelt, W. van, Asten, L. van, Kretzschmar, M., Schimmer, B., Nagelkerke, N.J.D., Vellema, P., Donker, G.A., Koopmans, M.P.G. In search of hidden Q-fever outbreaks: linking syndromic hospital clusters to infected goat farms. Epidemiology and Infection: 2011, 139(1), 19-26

\section{Hepatitis and endocarditis clusters}

Two hepatitis clusters (two signals) were detected in 2005-2007. The first was possibly due to hepatitis C [Appendix B, Table B2 (online)]. The other overlapped with reported abortion waves on goat farms, making Q fever its plausible cause. The cluster occurred more to the southeast (Fig. 3b) than the May/June 2007 outbreak. It would have given the first signal 2 weeks before the first reports of suspicious pneumonia patients in 2007. The cluster does not exceed the threshold level for highly significant clusters (i.e. recurrence interval 5 years).

For endocarditis, we revealed probable duplicates in all three detected clusters based on line-listing the age, postal code, and diagnoses of cluster patients. Although duplicates had been excluded from the analyses by using anonymous patient identifiers, some patients appeared to have obtained two or more anonymous identifiers through transfers between hospitals. We therefore excluded the endocarditis clusters from further analysis.

\section{DISCUSSION}

Using space-time syndromic surveillance methods, we found substantial support for the occurrence of human Q-fever outbreaks in The Netherlands preceding the 2007 outbreak and covering a wider area. In proximity to infected small-ruminant farms, local clusters of human LRI and hepatitis were detected in hospitalization data for 2005-2007. Although the retrospective nature of this study precluded laboratory confirmation, available epidemiological and surveillance data suggested Q fever as the cause for several syndrome clusters that mostly occurred in the southeast and middle of the country.

Prospective syndromic surveillance on hospitalizations, as simulated in this study, would have signalled some of these clusters long before detection of the May/June 2007 outbreak. The space-time signals of LRI or hepatitis could then have prompted further evaluation and/or laboratory tests, possibly confirming $C$. burnetii to be the causative pathogen. Although such efforts might not have prevented the large-scale outbreaks in the following years, it possibly would have lead to the detection and the appropriate treatment of Q-fever patients in a more timely manner. Apart from that, earlier detection of human Q-fever outbreaks might have facilitated research into transmission routes between specific farms and humans. For the Dutch human Q-fever outbreaks after 2007, this research has been complicated by dissemination of disease in the dairy-goat population, leading to widespread environmental contamination. These factors complicate the identification of specific farms as the source of human infections.

Of the LRI clusters detected in this study, one reflected the already known 2007 outbreak, confirming that syndromic surveillance can indeed detect Q-fever clusters, consistent with our findings on syndromic detection of respiratory outbreaks [13]. For six other LRI clusters and one hepatitis cluster as well, Q fever seemed a plausible cause. So far, hepatitis due to Q fever has been reported sporadically in The Netherlands: 33 hepatitis cases out of 1000 reported Q-fever cases in 2008 [5] and 5/178 in 2007. Hepatitis due to $Q$ fever might be more likely from infection by ingestion rather than inhalation $[25,26]$ and may thus follow distribution channels of contaminated unpasteurized products like raw cheese. If such contaminated products were to cause infections nationwide (without regional clustering), this would not be detected by our space-time analysis.

Our results suggest that the rise in reported Q-fever cases in 2008 and 2009 may partially reflect increased awareness among clinicians following the first recognized outbreak in 2007. This would be consistent with the fact that in 2008 and 2009, Q-fever reports originated from a wider area that overlaps with the area of most hospitalization clusters of 2005-2007 for which Q fever seemed a plausible cause. Moreover, the proportion of hospitalizations in confirmed Q-fever cases was smaller in 2008 and 2009 than in 2007, possibly indicating that increased awareness among clinicians led to diagnosis of earlier and milder Q-fever infections [5]. However, in 2007 we observed four clusters for which Q fever seemed a plausible cause vs. a total of three clusters in 2005 and 2006 combined, which suggests an actual rise in Q-fever infections in 2007.

Our finding that syndromic hospital data can reveal disease clusters possibly caused by Q fever, supports the value of prospective syndromic surveillance for detection of otherwise hidden outbreaks. We showed previously that syndromic surveillance for LRI would detect a modest number of clusters in time to investigate: on average five clusters per year at the lowest threshold level (recurrence intervals 1 year), and three clusters at the highest (recurrence intervals 5 years) [13]. If such prospective surveillance were to focus on detection of Q-fever outbreaks, inclusion of data on infected small-ruminant farms, as in the 
Wijngaard, C.C. van den, Dijkstra, F., Pelt, W. van, Asten, L. van, Kretzschmar, M., Schimmer, B., Nagelkerke, N.J.D., Vellema, P., Donker, G.A., Koopmans, M.P.G. In search of hidden Q-fever outbreaks: linking syndromic hospital clusters to infected goat farms. Epidemiology and Infection: 2011, 139(1), 19-26

current study, would further decrease the number of clusters to investigate. Nevertheless, even then such surveillance also requires sufficient complementary epidemiological and microbiological data to guide further investigation of detected clusters. For example, LRI clusters are best interpreted in relation to local influenza or RSV surveillance data. A prerequisite for prospective syndrome surveillance is the real-time availability of hospitalization data, including clinical diagnoses and symptoms by date of hospitalization. Although at present not available in The Netherlands, such real-time syndromic data collection might become feasible due to the nationwide implementation of electronic healthcare-information exchange. Finally, proper data collection and analysis will be more challenging in a prospective setting, for example due to data quality problems such as reporting delays.

This study has some further limitations. Due to its retrospective setting, C. burnetii infection could not be laboratory-confirmed for detected cluster patients. Therefore, we could only indicate whether Q fever seemed a plausible cause by excluding clusters with other apparent causes and assessing the presence of $C$. burnetii in cluster areas, as measured by overlap with infected farms. Another limitation lies in our use of voluntary reports from farmers to indicate possible $C$. burnetii point sources. Cluster areas not overlapping with reportedly infected farms may still have been contaminated by farms not tested or, possibly, by the spreading of manure from non-local contaminated farms. If so, this may explain those clusters that seemed possibly due to Q fever but did not overlap with reported Q fever on farms.

Serological testing of preserved human samples might confirm the occurrence of Q-fever clusters before 2007. However, to our knowledge no historical samples from specific LRI patients within the cluster areas are available. Other studies have found that substantial proportions of a human population can be exposed to $\mathrm{Q}$ fever without symptomatic infections [27, 28]. Therefore, a solution might be to use historical samples from blood donors, if sufficient samples from the cluster areas are still available.

This study shows substantial support for the occurrence of human Q-fever outbreaks in The Netherlands before detection of the May/June 2007 outbreak and covering a wider area. Retrospectively, suspicious LRI and hepatitis hospitalization clusters from 2005 to 2007 were detected and found to overlap with smallruminant farms reporting Q-fever abortion waves. Further research on historical serological samples from the detected cluster areas, if available, should be performed to confirm occurrence of human Q-fever outbreaks before the May/June 2007 outbreak. In a real-time setting, detection of these clusters should have prompted further investigation and additional laboratory tests, which might have resulted in detection of human Q-fever outbreaks up to 2 years earlier. In this light, it seems worthwhile to make syndromic hospitalization data available real-time for prospective outbreak detection. Finally, our study also illustrates the added value of integrated human and animal surveillance for improved detection of zoonotic disease outbreaks.

\section{ACKNOWLEDGEMENTS}

We thank Dutch Hospital Data for providing data from the Dutch National Medical Register (LMR); Daan Notermans and Marianne van der Sande for reviewing and commenting on the manuscript; and Lucy Phillips for editorial review.

\section{REFERENCES}

1. Raoult, D, Marrie, T, Mege, J. Natural history and pathophysiology of $Q$ fever. Lancet Infectious Diseases 2005; 5: 219-226.

2. Maurin, M, Raoult, D. Q fever. Clinical Microbiology Reviews 1999; 12: 518-553.

3. Carcopino, $X$, et al. Managing $Q$ fever during pregnancy: the benefits of long-term cotrimoxazole therapy. Clinical Infectious Diseases 2007; 45: 548-555.

4. van Gageldonk-Lafeber, $A B$, et al. Surveillance of $Q$ fever in the Netherlands [in Dutch]. Infectieziekten Bulletin 2003; 14: 173-177.

5. Schimmer, B, et al. Sustained intensive transmission of $Q$ fever in the south of the Netherlands, 2009. Eurosurveillance 2009; 14: pii=19210.

6. Karagiannis, I, et al. Investigation of a Q fever outbreak in a rural area of The Netherlands. Epidemiology and Infection 2009; 137: 1283-1294.

7. Schimmer, B, et al. Large ongoing $Q$ fever outbreak in the south of The Netherlands, 2008.

Eurosurveillance 2008; 13: pii=18939. 
Wijngaard, C.C. van den, Dijkstra, F., Pelt, W. van, Asten, L. van, Kretzschmar, M., Schimmer, B., Nagelkerke, N.J.D., Vellema, P., Donker, G.A., Koopmans, M.P.G. In search of hidden Q-fever outbreaks: linking syndromic hospital clusters to infected goat farms. Epidemiology and Infection: 2011, 139(1), 19-26

8. Van Steenbergen, JE, et al. An outbreak of $Q$ fever in The Netherlands - possible link to goats [in Dutch]. Nederlands Tijdschrift voor Geneeskunde 2007; 151: 1998-2003.

9. Wouda, W, Dercksen, DP. Abortion and stillbirth among dairy goats as a consequence of Coxiella burnetii [in Dutch]. Tijdschrift voor Diergeneeskunde 2007; 132: 908-911.

10. Morroy, G, et al. Q-fever: will the mystery be solved? [in Dutch]. Infectieziekten Bulletin 2008; 19: 6668.

11. Lazarus, $R$, et al. Using automated medical records for rapid identification of illness syndromes (syndromic surveillance): the example of lower respiratory infection. BMC Public Health 2001; 1: 9.

12. Heffernan, R, et al. Syndromic surveillance in public health practice, New York City. Emerging Infectious Diseases 2004; 10: 858-864.

13. van den Wijngaard, $\mathrm{CC}$, et al. Syndromic surveillance for local outbreaks of lower-respiratory infections: would it work? PLoS ONE 2010; 5: e10406.

14. Olson, KL, Grannis, SJ, Mandl, KD. Privacy protection versus cluster detection in spatial epidemiology. American Journal of Public Health 2006; 96: 2002-2008.

15. Higgs, BW, et al. Early detection of tuberculosis outbreaks among the San Francisco homeless: tradeoffs between spatial resolution and temporal scale. PLoS ONE 2007; 2: e1284.

16. Donker, GA. Continuous morbidity registration sentinels Netherlands 2006 [in Dutch]. NIVEL, Annual Report, 2007.

17. Rousset, $E$, et al. Coxiella burnetii shedding routes and antibody response after outbreaks of $Q$ feverinduced abortion in dairy goat herds. Applied and Environmental Microbiology 2009; 75: 428-433.

18. Kulldorff, M, et al. A space-time permutation scan statistic for disease outbreak detection. PLoS Med 2005; 2: e59.

19. Kulldorff, M, and Information Management Services Inc. SaTScanTM v7.0: Software for the spatial and space-time scan statistics, 2006. Accessed 18 March 2008.

20. Abrams, AM, Kleinman, KP. A SaTScan macro accessory for cartography (SMAC) package implemented with SAS software. International Journal of Health Geographics 2007; 6: 6.

21. Kleinman, K, Lazarus, R, Platt, R. A generalized linear mixed models approach for detecting incident clusters of disease in small areas, with an application to biological terrorism. American Journal of Epidemiology 2004; 159: 217-224.

22. Kulldorff, M. Prospective time periodic geographical disease surveillance using a scan statistic. Journal of the Royal Statistical Society A 2001; 164: 61-72.

23. Gilsdorf, A, et al. Large $Q$ fever outbreak due to sheep farming near residential areas, Germany, 2005. Epidemiology and Infection 2008; 136: 1084-1087.

24. Van den Hoek, JA, IJzerman, EP, Coutinho, RA. Legionella outbreak in Amsterdam: a cooling tower as the source [in Dutch]. Nederlands Tijdschrift voor Geneeskunde 2006; 150: 1808-1811.

25. La Scola, B, Lepidi, H, Raoult, D. Pathologic changes during acute $Q$ fever: influence of the route of infection and inoculum size in infected guinea pigs. Infection \& Immunity 1997; 65: 2443-2447.

26. Marrie, TJ, et al. Route of infection determines the clinical manifestations of acute $Q$ fever. Journal of Infectious Diseases 1996; 173: 484-487.

27. Marrie, TJ, Pollak, PT. Seroepidemiology of $Q$ fever in Nova Scotia: evidence for age dependent cohorts and geographical distribution. European Journal of Epidemiology 1995; 11: 47-54.

28. McCaughey, C, et al. Human seroprevalence to Coxiella burnetii ( $Q$ fever) in Northern Ireland. Zoonoses Public Health 2008; 55: 189-194.

\section{FIGURES}


Wijngaard, C.C. van den, Dijkstra, F., Pelt, W. van, Asten, L. van, Kretzschmar, M., Schimmer, B., Nagelkerke, N.J.D., Vellema, P., Donker, G.A., Koopmans, M.P.G. In search of hidden Q-fever outbreaks: linking syndromic hospital clusters to infected goat farms. Epidemiology and Infection: 2011, 139(1), 19-26

STEP 1

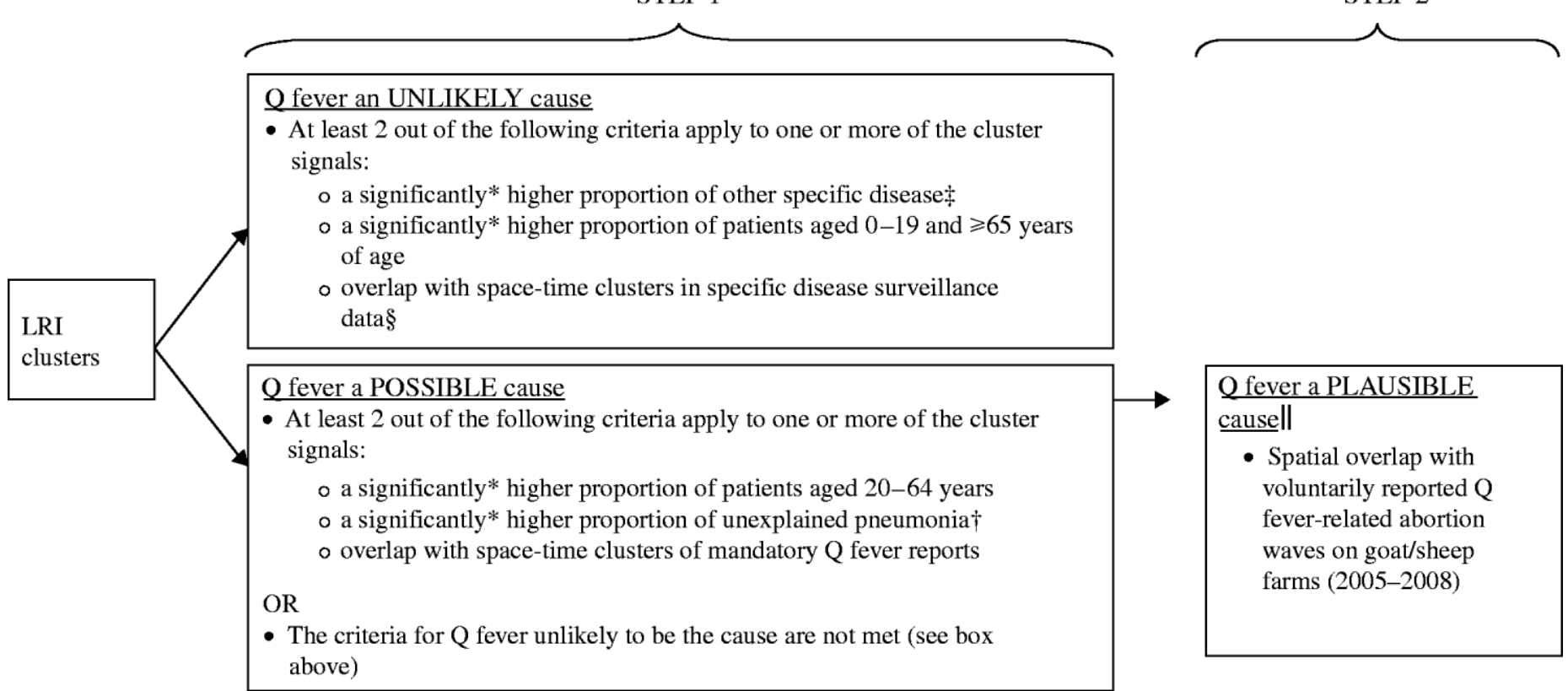

Fig. 1. Two-step criteria to explore the plausibility that $Q$ fever caused the lower-respiratory infection (LRI) hospitalization clusters detected in $2005-2007$. * Right-sided Fisher's exact test for $2 \times 2$ tables $(\alpha \leqslant 0 \cdot 05$ and/or <0.01) of hospitalizations inside vs. outside of the cluster signal. The proportion of hospitalizations with a specific disease characteristic (e.g. Legionnaires' disease as discharge diagnoses, or patients aged 20-64 years) can be significantly higher in hospitalizations within the cluster signal than the proportion outside the cluster signal. † ICD-9 codes 485/486/481/4829. $¥$ We considered high proportions of bronchitis/bronchiolitis (ICD-9 codes 4801/4660/4661/490) as a likely indication for RSV activity. $\S$ Mandatory reports and influenza-like illness sentinel data. || Only assessed if the cluster meets the criteria for $Q$ fever to be a possible cause.

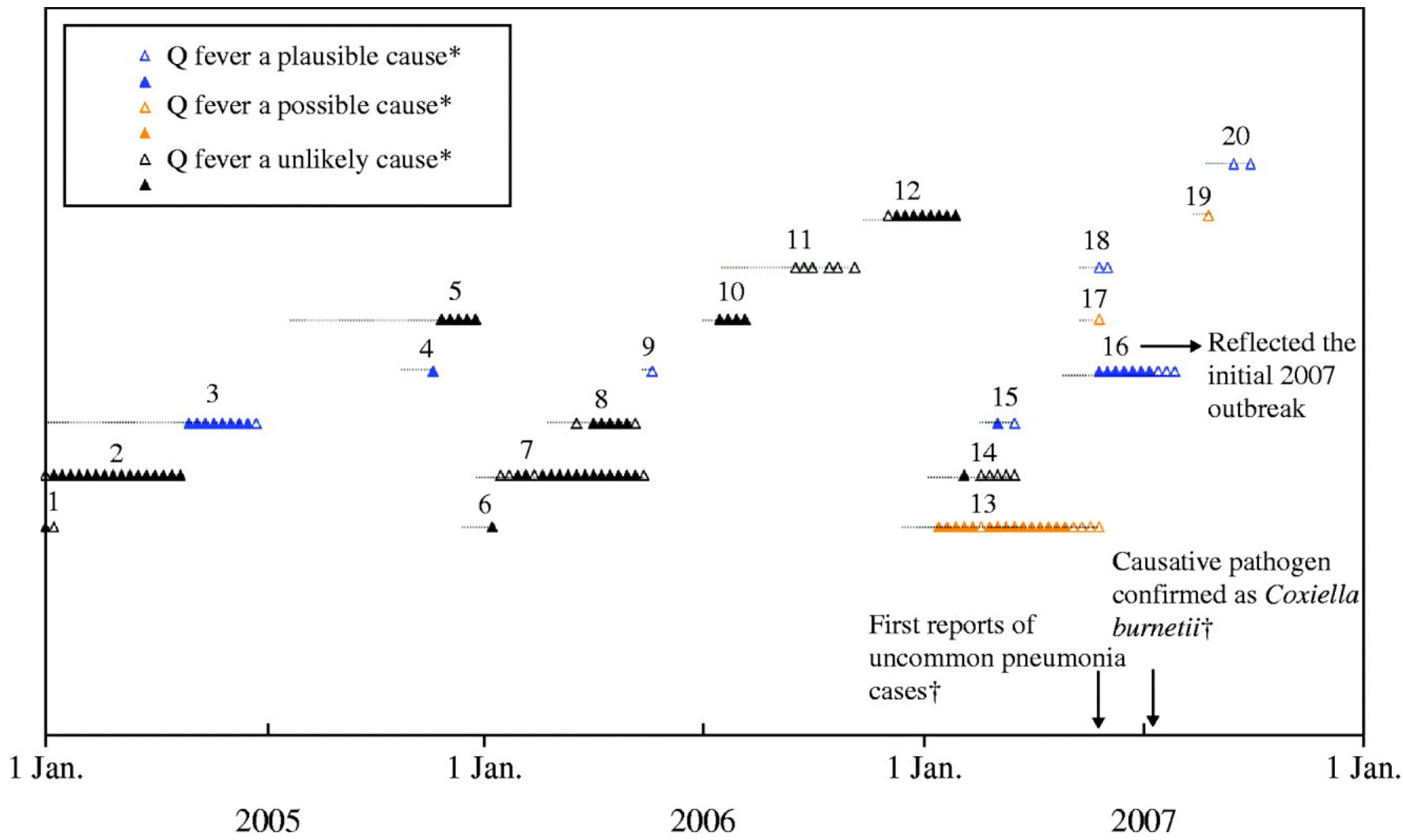


Wijngaard, C.C. van den, Dijkstra, F., Pelt, W. van, Asten, L. van, Kretzschmar, M., Schimmer, B., Nagelkerke, N.J.D., Vellema, P., Donker, G.A., Koopmans, M.P.G. In search of hidden Q-fever outbreaks: linking syndromic hospital clusters to infected goat farms. Epidemiology and Infection: 2011, 139(1), 19-26

Fig. 2. All lower-respiratory infection (LRI) signals and clusters on a timescale. The legend indicates which clusters have $Q$ fever as a plausible, possible, or unlikely cause (see criteria in Fig. 1). * For each cluster, horizontal dotted lines indicate the total cluster episode, and triangles indicate weekly generated cluster signals. As some clusters overlapped in time, consecutive clusters are presented at different heights at the $y$-axis. The significance level of cluster signals, as measured by the recurrence interval (see Methods section), is indicated by the colour value of the triangles. For those with uncoloured backgrounds, the recurrence interval of the signal is $\geqslant 1$ year; with coloured backgrounds, the recurrence interval is $\geqslant 5$ years). † At the end of May 2007 the first uncommon pneumonia patients were reported in the area of the initial 2007 outbreak, and on 11 July C. burnetii was confirmed to be the causative pathogen. This initial 2007 outbreak was reflected by cluster 16 .

(a)

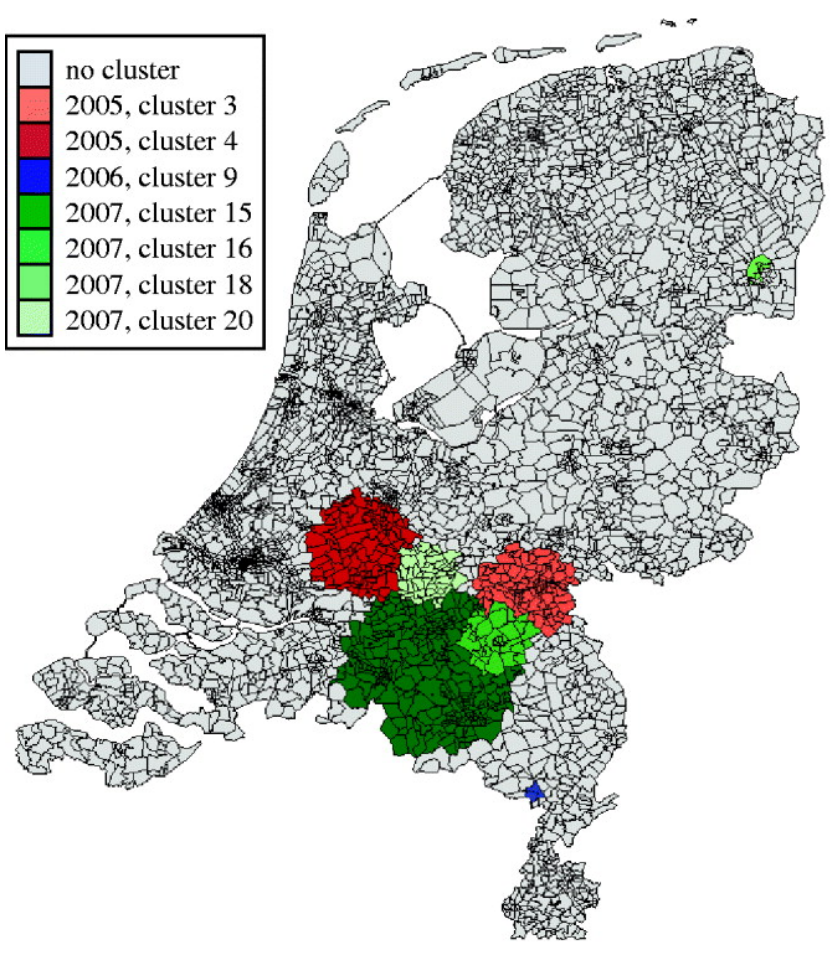

(b)

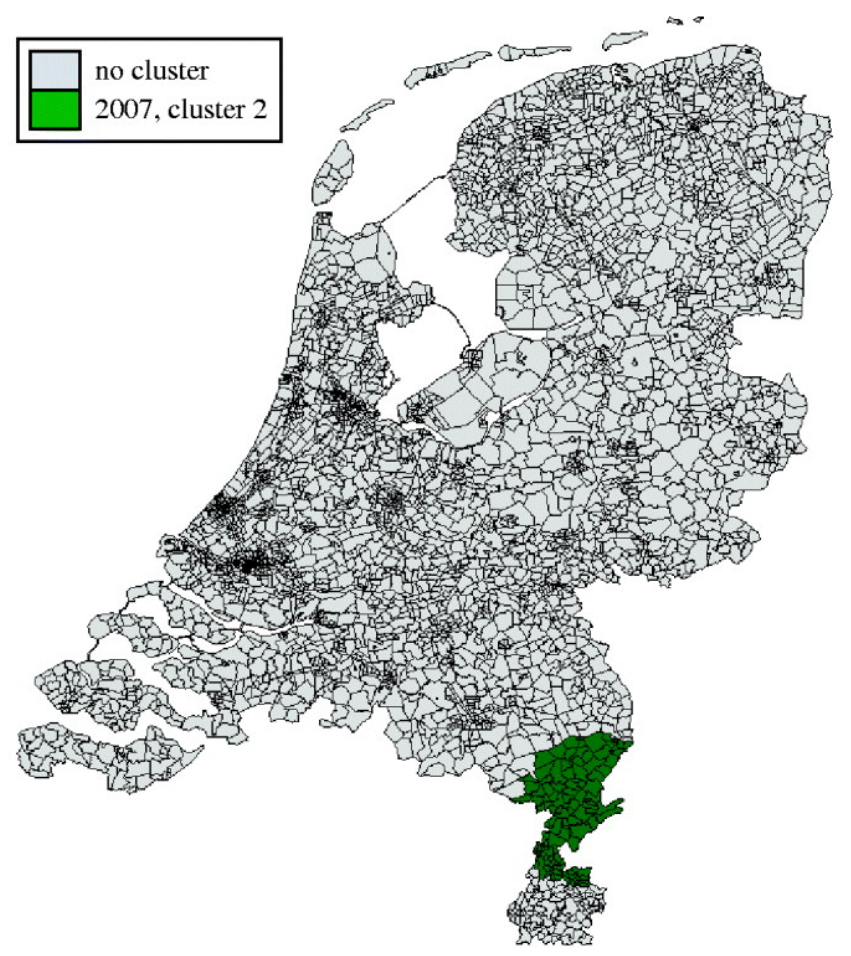

Fig. 3. (a) Lower-respiratory infection (LRI) clusters for which $Q$ fever seemed a plausible cause, presented on the 4-digit postal code map of The Netherlands. See criteria for $Q$ fever as a plausible cause in Figure 1. If LRI cluster areas overlapped, the smaller cluster area was drawn on top of the larger area. (b) The hepatitis cluster for which $Q$ fever seemed a plausible cause, presented on the 4-digit postal code map of The Netherlands. 